TITLE:

\title{
Anomalous behavior of the coefficient of normal restitution in oblique impact
}

$\operatorname{AUTHOR}(S)$ :

Kuninaka, $\mathrm{H}$; Hayakawa, $\mathrm{H}$

\section{CITATION:}

Kuninaka, $\mathrm{H}$... [et al]. Anomalous behavior of the coefficient of normal restitution in oblique impact. PHYSICAL REVIEW LETTERS 2004, 93(15): 154301.

ISSUE DATE:

2004-10-08

URL:

http://hdl.handle.net/2433/50060

RIGHT:

Copyright 2004 American Physical Society 


\title{
Anomalous Behavior of the Coefficient of Normal Restitution in Oblique Impact
}

\author{
Hiroto Kuninaka ${ }^{1, *}$ and Hisao Hayakawa ${ }^{2}$ \\ ${ }^{1}$ Graduate School of Human and Environmental Studies, Kyoto University, Sakyo-ku, Kyoto, Japan, 606-8501 \\ ${ }^{2}$ Department of Physics, Yoshida-south campus, Kyoto University, Sakyo-ku, Kyoto, Japan, 606-8501
}

(Received 3 October 2003; published 5 October 2004)

\begin{abstract}
The coefficient of normal restitution in an oblique impact is theoretically studied. Using a twodimensional lattice model for an elastic disk and an elastic wall, we demonstrate that the coefficient of normal restitution can exceed unity and has a peak against the incident angle in our simulation. We also explain this behavior based upon a phenomenological theory.
\end{abstract}

DOI: 10.1103/PhysRevLett.93.154301

The coefficient of normal restitution $e$ is introduced to determine the normal component of the postcollisional velocity in the collision of two materials. The coefficient $e$ is defined by

$$
\mathbf{v}\left(\tau_{c}\right) \cdot \mathbf{n}=-e \mathbf{v}(0) \cdot \mathbf{n},
$$

where $\mathbf{v}(\tau)$ is the relative velocity of the centers of mass of two colliding materials at time $\tau$ measured from the initial contact, $\tau_{c}$ is the duration of a collision, and $\mathbf{n}$ is the unit vector normal to the contact plane. Though some text books of elementary physics state that $e$ is a material constant, many experiments and simulations show that $e$ decreases with increasing impact velocity [1]. The dependence of $e$ on the low impact velocity is theoretically treated by the quasistatic theory [2-4]. We also recognize that $e$ can be less than unity for the normal impact without the introduction of any explicit dissipation, because the macroscopic inelasticities originate in the transfer of the energy from the translational mode to the internal modes such as the vibrations $[3,5,6]$.

While $e$ has been believed to be less than unity in most situations, it is recently reported that $e$ can exceed unity in oblique impacts [7-9]. In particular, Louge and Adams [9] observed oblique impacts of a hard aluminum oxide sphere on a thick elastoplastic polycarbonate plate and found that $e$ grows monotonically with the magnitude of the tangent of the incident angle $\gamma$. In their experiment, Young's modulus of the plate is 100 times smaller than that of the aluminum oxide sphere. They also suggested that $e$ can exceed unity for the most oblique impacts.

In this Letter, we demonstrate that our twodimensional simulation of the oblique impact based on Hamilton's equation has yielded an increasing $e$ with $\tan \gamma$ and $e$ exceeds unity at the critical incident angle. Finally, we explain our results by our phenomenological theory.

Let us introduce our numerical model [10]. Our model consists of an elastic disk and an elastic wall (Fig. 1). The width and the height of the wall are $8 R$ and $2 R$, respectively, where $R$ is the mean radius of the undeformed disk. Both side ends and the bottom of the wall are fixed. We
PACS numbers: 45.50.Tn, 45.70.-n, 62.20.-x, 81.40.Pq

place 800 mass points at random in a disk with the radius $R$ and 4000 mass points at random in a wall for the disk and the wall, respectively. We connect each mass point with its neighbor mass points by the Delaunay triangulation algorithm [11], and undeformed nonlinear springs are placed on all the connections.

Each mass point $i$ on the lower half boundary of the disk feels the force, $\mathbf{F}\left(l_{s}^{(i)}\right)=a V_{0} \exp \left(-a l_{s}^{(i)}\right) \mathbf{n}_{s}^{(i)}$, where $l_{s}^{(i)}$ is the distance between the $i$ th surface mass point of the disk and the nearest surface spring of the wall, $a=$ $300 / R, V_{0}=a m c^{2} R / 2, m$ is the mass of each mass point, $c$ is the one-dimensional velocity of sound, and $\mathbf{n}_{s}^{(i)}$ is the unit vector normal to the connection between two surface mass points of the wall [10]. We should note that the strong repulsion $\mathbf{F}\left(l_{s}^{(i)}\right)$ is introduced to inhibit the penetration of the disk to the surface of the wall [5]. Thus, the dynamical equation of motion for each mass point $i$ of the lower half boundary of the disk is described by

$$
\begin{aligned}
m \frac{d^{2} \mathbf{r}_{i}}{d \tau^{2}}= & \sum_{j=1}^{N_{i}}\left\{-k_{a} \mathbf{x}_{i j}-k_{b} \mathbf{x}_{i j}^{3}\right\}+\Theta\left(l_{t h}\right. \\
& \left.-l_{s}^{(i)}\right) a V_{0} \exp \left(-a l_{s}^{(i)}\right) \mathbf{n}_{s}^{(i)},
\end{aligned}
$$

where $\mathbf{r}_{i}$ is the position of $i$ th mass point, $N_{i}$ is the number of mass points connected to the $i$ th mass point, $\mathbf{x}_{i j}$ is the

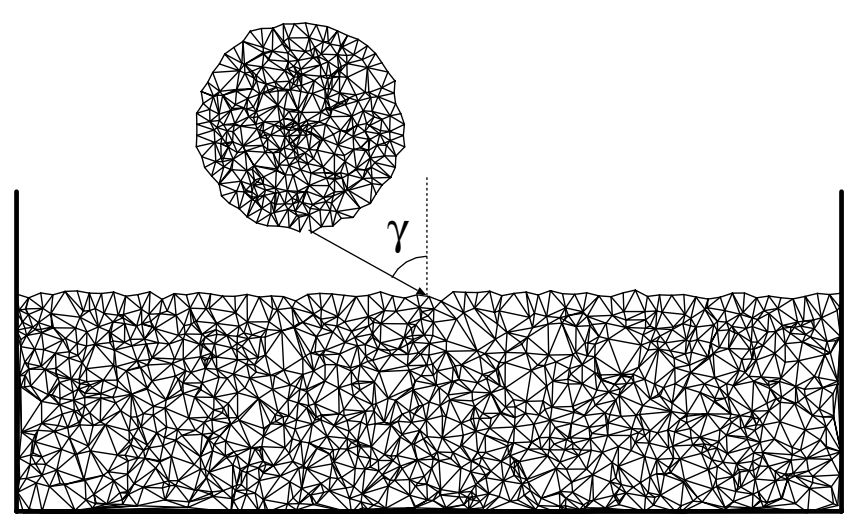

FIG. 1. The elastic disk and wall consisted of random lattice. 
relative deformation of the spring between $i$ th and $j$ th connected mass points, and $k_{a}$ and $k_{b}=k_{a} \times 10^{-3} / R^{2}$ are the spring constants. Here we introduce the step function $\Theta(x)$, i.e., $\Theta(x)=1$ for $x \geq 0$ and $\Theta(x)=0$ for $x<0$, and the threshold length $l_{t h}$ which is the average of the natural lengths of the springs of the disk. For internal mass points, the last term of the right hand side of Eq. (2) is omitted. In most of our simulations, we adopt $k_{a}=k_{a}^{(d)}=1.0 \times m c^{2} / R^{2}$ for the disk and $k_{a}=k_{a}^{(w)}=$ $1.0 \times 10^{-2} m c^{2} / R^{2}$ for the wall. We do not introduce any dissipative mechanism in this model. Thus, during a collision, a part of the initial translational energy of the disk is distributed into the vibrational energy of the disk and the wall. It should be noted that the macroscopic dissipation can be interpreted as the irreversible transfer of the energy from the translational motion to the internal vibration. When we introduce explicit dissipations in the model and add the gravity to the disk, we have confirmed that the compression of the disk can be described by twodimensional Hertzian contact theory [12].

In this model, the roughness of the surfaces is important to make the disk rotate after a collision [10]. We modify the positions of the surface mass points of the flat wall and the smooth disk by using normal random numbers whose average and standard deviation are 0 and $3 \times$ $10^{-2} R$, respectively.

Poisson's ratio $\nu$ and Young's modulus $E$ of this model can be evaluated by adding the external force to stretch the rectangle of random lattice numerically. We obtain Poisson's ratio $\nu=(7.50 \pm 0.11) \times 10^{-2}$ and Young's modulus $E=(9.54 \pm 0.231) \times 10^{3} m c^{2} / R^{2}$, respectively [10].

We solve the dynamical equation of motion (2) for each mass point with the initial speed $|\mathbf{v}(0)|=0.1 c$ and the incident angle $\gamma$, and determine $e$ for each $\gamma$ according to Eq. (1). All the results in this Letter are obtained by averaging the results of 100 disks with different configu-

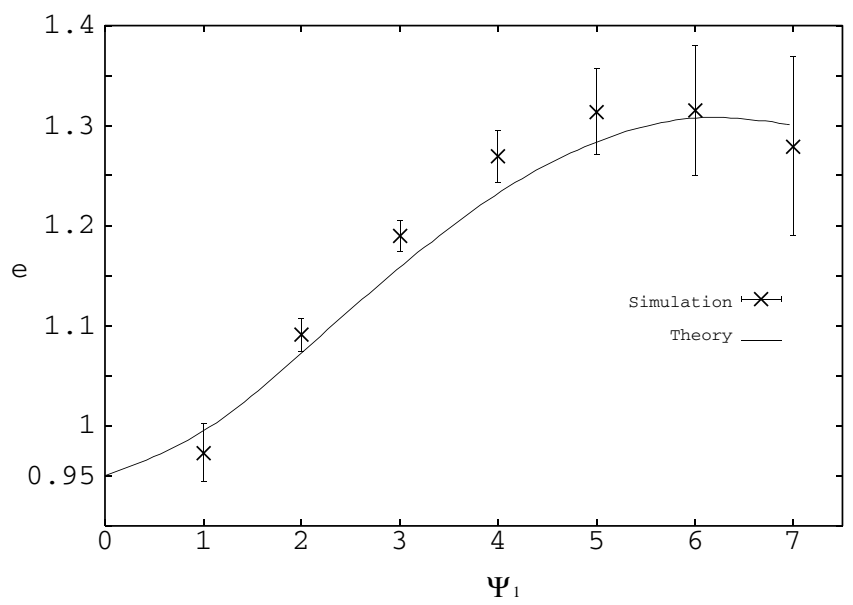

FIG. 2. Numerical and theoretical results of the relation between $\Psi_{1}$ and $e$. rations of mass points. We use the fourth order symplectic integrator with the time step $\Delta \tau=10^{-3} R / c$.

Figure 2 is the relation between $e$ and $\Psi_{1} \equiv-(\mathbf{v}(0)$. $\mathbf{t}) /(\mathbf{v}(0) \cdot \mathbf{n})=\tan \gamma$, where $\mathbf{t}$ is the unit vector vertical to n. The cross point is the mean value and the error bar is the standard deviation of 100 samples for each $\gamma$. This result shows that $e$ increases with increasing $\Psi_{1}$ to exceed unity, and has a peak around $\Psi_{1}=6.0$. The behavior of $e$ having the peak is in contrast to that in the experiment by Louge and Adams [9].

Here, let us explain our results. Louge and Adams [9] suggest that their results can be explained by the rotation of the normal unit vector $\mathbf{n}$ arising from the local deformation of the wall's surface. Thus, we aim to determine the angle of rotation of the unit vector $\alpha$ at each $\gamma$ from the theory of elasticity.

Figure 3 is the schematic figure of a hard disk moving from the left to the right on a wall, where $l \equiv\left|x_{b}-x_{a}\right|$. Assuming that $l$ is small compared to $R$, the perimeter of the contact is approximated by

$$
f(x)=\left(x-x_{c}\right)^{2} / 2 R-y_{c},
$$

where $\left(x_{c}, y_{c}\right)$ is the lowest position of the disk. To calculate $\tan \alpha \equiv\left(f\left(x_{b}\right)-f\left(x_{a}\right)\right) / l$, we need to know the ratio of $\left|x_{c}-x_{a}\right|$ to $l$. From the theory of elasticity [13], this ratio can be estimated as

$$
\frac{x_{c}-x_{a}}{l}=1-\theta \quad \text { with } \theta=\frac{1}{\pi} \arctan \frac{1-2 \nu}{\mu(2-2 \nu)},
$$

where $\mu$ is the coefficient of the friction. We evaluate $\mu$ by $\mu \equiv|\mathbf{J} \cdot \mathbf{t}| /|\mathbf{J} \cdot \mathbf{n}|$ with $\mathbf{J}=M(\mathbf{v}(\tau)-\mathbf{v}(0))$, where $M$ is the mass of the disk. The cross points in Fig. 4 represent $\mu$ calculated from our simulation, where $\mu$ has a peak around $\Psi_{1}=3.0$. From Eqs. (3) and (4), $\tan \alpha$ is given by

$$
\tan \alpha=\frac{2 \theta-1}{2-2 \theta} \frac{\left|x_{c}-x_{a}\right|}{R},
$$

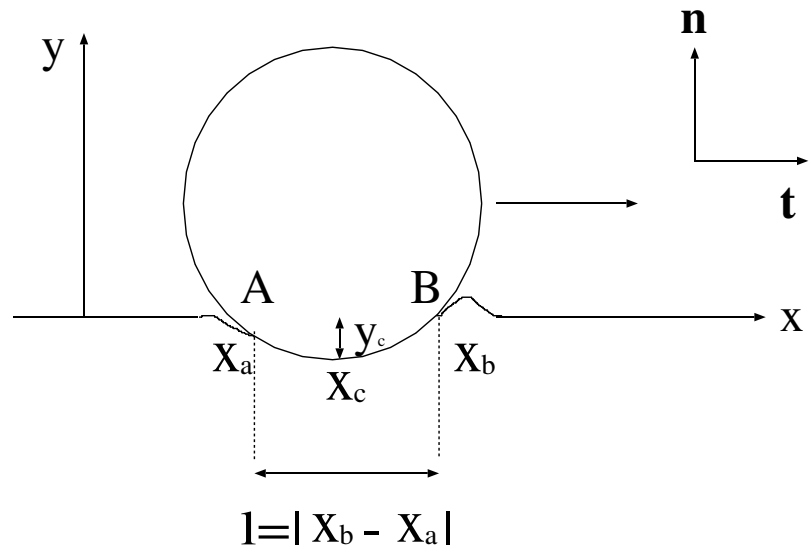

FIG. 3. The schematic figure of a hard disk sliding on a soft wall. $x$ coordinates of both ends of the contact area $A B$ are $x=$ $x_{a}$ and $x=x_{b}$. 


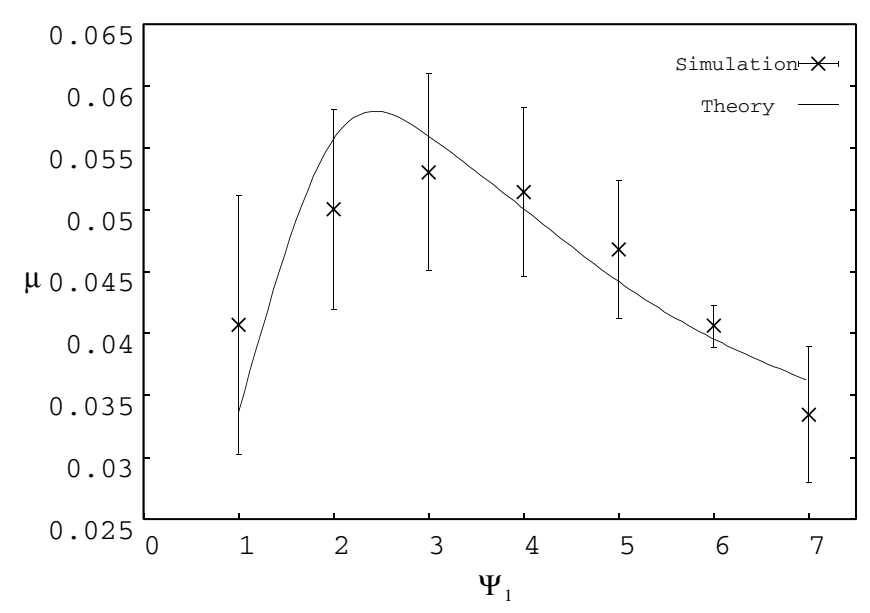

FIG. 4. Numerical and theoretical results of the relation between $\Psi_{1}$ and $\mu$.

where we evaluate $\left|x_{c}-x_{a}\right|=0.55 R$ from the maximum value of the compression of the disk. From Eqs. (4) and (5), we obtain the relation between $\Psi_{1}$ and $\tan \alpha$.

Next, let us calculate $e$ from the relation between $\Psi_{1}$ and $\tan \alpha$. We introduce the rotated unit vectors, $\mathbf{n}_{\alpha}$ and $\mathbf{t}_{\alpha}$, as $\mathbf{n}_{\alpha}=\cos \alpha \mathbf{n}-\sin \alpha \mathbf{t}$ and $\mathbf{t}_{\alpha}=\sin \alpha \mathbf{n}+\cos \alpha \mathbf{t}$, respectively. By introducing $e_{\alpha} \equiv-\left(\mathbf{v}\left(\tau_{c}\right) \cdot \mathbf{n}_{\alpha}\right) /(\mathbf{v}(0)$. $\mathbf{n}_{\alpha}$ ), we can express $e$ in terms of $e_{\alpha}$ as

$$
e=\frac{e_{\alpha}+\Psi_{2}^{\alpha} \tan \alpha}{1-\Psi_{1}^{\alpha} \tan \alpha}
$$

where $\Psi_{1}^{\alpha}=-\left(\mathbf{v}(0) \cdot \mathbf{t}_{\alpha}\right) /\left(\mathbf{v}(0) \cdot \mathbf{n}_{\alpha}\right)$ and $\Psi_{2}^{\alpha}=-\left(\mathbf{v}\left(\tau_{c}\right)\right.$. $\left.\mathbf{t}_{\alpha}\right) /\left(\mathbf{v}(0) \cdot \mathbf{n}_{\alpha}\right) . \Psi_{1}^{\alpha}$ also can be rewritten as

$$
\Psi_{1}^{\alpha}=\left(\Psi_{1}-\tan \alpha\right) /\left(1+\Psi_{1} \tan \alpha\right) .
$$

On the other hand, in the oblique impact of slipping disks, $\Psi_{2}^{\alpha}$ is given by

$$
\Psi_{2}^{\alpha}=\Psi_{1}^{\alpha}-3\left(1+e_{\alpha}\right) \mu_{\alpha}
$$

in the two-dimensional situation [14]. In Eq. (8), $\mu_{\alpha}$, defined by $\mu_{\alpha}=\left|\mathbf{J} \cdot \mathbf{t}_{\alpha}\right| /\left|\mathbf{J} \cdot \mathbf{n}_{\alpha}\right|$, is given by

$$
\mu_{\alpha}=\frac{\mu+\tan \alpha}{1-\mu \tan \alpha} \text {. }
$$

To draw the solid line in Fig. 2, at first, we calculate $\mu$ and $\tan \alpha$ for each $\Psi_{1}$. By choosing a fitting parameter $e_{\alpha}=$ 0.95 and substituting Eqs. (7)-(9) into Eq. (6) we obtain $e$ as a function of $\alpha, \mu$, and $\Psi_{1}$. All points are interpolated with the cubic spline interpolation method to draw the solid curve. This theoretical description of $e$ is qualitatively consistent with our numerical result, though the theoretical value is a little smaller than the observed value.

Now, let us consider how $\mu$ depends on $\Psi_{1}$ based on a phenomenological argument. For simplicity, we replace the roughness on the surface of the wall by a periodic array of asperities. When the disk hits one asperity, a fraction of the energy is absorbed in the wall. We assume that the tangential velocity $v_{t}^{(i-1)}$ changes to $v_{t}^{(i)}=(1-$ $\eta) v_{t}^{(i-1)}$ when the disk hits the $i$ th asperity. Thus, if the disk contacts $N$ asperities during the impact, the tangential speed at the release point becomes $v_{t}\left(\tau_{c}\right)=v_{t}^{(N)}=$ $(1-\eta)^{N} v_{t}(0)$. Here we estimate the number of contacted asperities during the collision as $N=\rho l_{s l}$, where $\rho$ is the number of the asperities in a unit length on the surface and $l_{s l}$ is the length of sliding which can be evaluated as $l_{s l}=v_{t}(0) \tau_{c}$ with $\tau_{c}=\pi(R / c) \sqrt{\ln \left[4 c / v_{n}(0)\right]}$ [6]. Thus, the tangential impulse $J_{t} \equiv \mathbf{J} \cdot \mathbf{t}$ is approximated by

$$
J_{t}=M\left\{(1-\eta)^{\rho l_{s l}}-1\right\} v_{t}(0) \simeq-\eta M \rho l_{s l} v_{t}(0),
$$

where we assume small $\eta$. Now, we should answer the question how to determine $\eta$. When the impact velocity is large enough, $\eta$ becomes smaller because the asperities are broken down when the disk hits them. Therefore we may assume the form $\eta=\eta_{0} /\left(1+\beta v_{t}^{2}(0) / v_{n}^{2}(0)\right)=$ $\eta_{0} /\left(1+\beta \Psi_{1}^{2}\right)$ with the introduction of the dimensionless parameters $\eta_{0}$ and $\beta$. Taking into account $J_{n} \equiv \mathbf{J} \cdot \mathbf{n}=$ $-M(1+e) v_{n}(0)$ and the definition of $\mu=J_{t} / J_{n}$, we obtain

$$
\mu=\frac{\pi \eta_{0} \rho R}{1+e} \frac{\Psi_{1}}{1+\beta \Psi_{1}^{2}} \sqrt{\frac{\Psi_{1}^{2}}{1+\Psi_{1}^{2}} \ln \left(40 \sqrt{1+\Psi_{1}^{2}}\right)} \frac{|\mathbf{v}(0)|}{c} .
$$

Here we use $\cos \gamma=\sqrt{1 /\left(1+\Psi_{1}^{2}\right)}, \quad \sin \gamma=$ $\sqrt{\Psi_{1}^{2} /\left(1+\Psi_{1}^{2}\right)}$. We adopt the numerical results for the value of $e$ at each $\Psi_{1}$ (Fig. 2) and $|\mathbf{v}(0)| / c=0.1$. The solid curve in Fig. 4 is Eq. (11) with fitting parameters $\beta=0.21$ and $\eta_{0} \rho R=0.18$, which reproduces our numerical result. We do not claim that our simple argument explains the experimental result because of two fitting parameters. However, we emphasize that our picture captures the essence of the behavior of $\mu$ in the oblique impact.

Let us discuss our result. First, we emphasize that the novel phenomena of $e$ exceeding unity are obtained from the local deformation of the soft wall for the oblique impacts of a hard disk. When we simulate the impact between a disk and a hard wall, for $k_{a}^{(w)}=10 \times k_{a}^{(d)}, e$ fluctuates around a constant to exceed unity abruptly around $\Psi_{1}=4.5$ [12]. This tendency resembles the experimental results by Calsamiglia et al. [8]. Thus, for a smooth increase of $e$ to exceed unity, the wall should be softer than the disk. In addition, it is important to fix the initial kinetic energy of the disk. So far, we have confirmed that $e$ does not exceed unity when $\Psi_{1}$ is controlled by changing $v_{t}$ with fixed $v_{n}$ [10].

Second, the initial velocity of the disk and the local deformation of the wall are much larger than those in the experimental ones in Ref. [9]. They cause the most sig- 
nificant difference between our result and their result [9]. Because of the high speed impact in our case, there is a peak of $e$ for small $\gamma$. In fact, our simulation with $|\mathbf{v}(0)|=$ $0.01 c$ shows the shift of the peak for larger $\gamma$. Therefore, we expect that our model reproduces the result of Ref. [9] for the low impact speed. In addition, we have carried out simulations with a disk of 400 mass points and a wall of 2000 mass points to check the effect of the system size. Although there is a slight difference between the results, the data are also well reproducible by our phenomenological theory.

Third, the local deformation of the wall also affects the relation between $\mu$ and $\Psi_{1}$. In early studies, it has been shown that $\mu$ depends on the impact velocity $[9,15]$. In our simulation, $\mu$ has a peak around $\Psi_{1}=3.0$. This behavior is interpreted as that the asperities are flattened for large $v_{t}$. Equation (11) indicates that $\mu$ can increase with increasing $\gamma$ as in Ref. [9] if we choose a suitable set of $\beta$ and $\eta_{0} \rho$. The difference between the results of their experiment and our simulation may be explained by the choice of these parameters.

Fourth, we adopt the static theory of elasticity to explain our numerical results in this Letter. However, it is important to solve the time-dependent equation of the deformation of the wall's surface to analyze the dynamics of impact phenomena. The dynamical analysis will be our future task.

Finally, we indicate that the friction coefficient is derived from our Hamiltonian model. This friction comes from the irreversible energy transfer of the macroscopic translational motion to the internal vibration. The irreversibility is indeed related to the second law of thermodynamics if the number of the internal degrees of freedom is infinite. However, the irreversibility in our system which includes only $10^{3}$ mass points in a disk is incomplete as we can see that the Hertzian contact theory cannot be recovered without introduction of the explicit dissipation. We believe that the complete treatment of the inelastic collision of macroscopic materials without introduction of dissipations will be a fundamental subject of nonequilibrium statistical mechanics.

In summary, we have carried out the two-dimensional simulation of the oblique impact of an elastic disk on an elastic wall. We have found that $e$ can exceed unity in the oblique impact, which is attributed to the local deformation of the wall. We have estimated the magnitude of the local deformation $\alpha$ based on the static theory of elasticity and derived the relation between $e$ and $\Psi_{1}$ by taking into account the rotation of the normal unit vector of the wall's surface. The relation between $\mu$ and $\Psi_{1}$ is also related to the local deformation and is explained by the simple argument.

We thank Y. Tanaka and S. Nagahiro for their valuable comments. We also thank M. Y. Louge for his detailed introduction of their experiment to us and some valuable comments. Parts of the numerical computation in this work were carried out at Yukawa Institute Computer Facility. This study is partially supported by the Grantin-Aid of Ministry of Education, Culture, Sports, Science and Technology (MEXT), Japan (Grant No. 15540393), and the Grant-in-Aid for the 21st century COE "Center for Diversity and Universality in Physics" from MEXT, Japan.

*Present address: Department of Chemistry, Kyoto University, Sakyo-ku, Kyoto, Japan, 606-8502.

Email address: kuninaka@kuchem.kyoto-u.ac.jp

[1] W. J. Stronge, Impact Mechanics (Cambridge University Press, Cambridge, 2000).

[2] G. Kuwabara and K. Kono, Jpn. J. Appl. Phys. 26, 1230 (1987).

[3] W. A. M. Morgado and I. Oppenheim, Phys. Rev. E 55, 1940 (1997).

[4] N.V. Brilliantov, F. Spahn, J.-M. Hertzsch, and T. Pöschel, Phys. Rev. E 53, 5382 (1996).

[5] F. Gerl and A. Zippelius, Phys. Rev. E 59, 2361 (1999).

[6] H. Hayakawa and H. Kuninaka, Chem. Eng. Sci. 57, 239 (2002).

[7] C. E. Smith and P.-P. Liu, J. Appl. Mech. 59, 963 (1992).

[8] J. Calsamiglia, S.W. Kennedy, A. Chatterjee, A. Ruina, and J. T. Jenkins, J. Appl. Mech. 66, 146 (1999).

[9] M. Y. Louge and M. E. Adams, Phys. Rev. E 65, 021303 (2002).

[10] H. Kuninaka and H. Hayakawa, J. Phys. Soc. Jpn. 72, 1655 (2003).

[11] K. Sugihara, Data Structure and Algorithms (Kyoritsu, Japan, 2001) (in Japanese).

[12] H. Kuninaka, Ph.D. thesis, Kyoto University, 2004; H. Hayakawa and H. Kuninaka, Phase Transit. 77, 889 (2004).

[13] L. A. Galin, Contact Problems in the Theory of Elasticity (Gostekhizdat, Moskow, 1953).

[14] O. R. Walton and R. L. Braun, J. Rheol. (N.Y.) 30, 949 (1986).

[15] D. A. Gorham and A. H. Kharaz, Powder Technol. 112, 193 (2000). 